\title{
The effects of framing on decision making: Collaborative versus individual decision making among older adults
}

\author{
Sarah A. Stoner \\ West Virginia University
}

Follow this and additional works at: https://researchrepository.wvu.edu/etd

\section{Recommended Citation}

Stoner, Sarah A., "The effects of framing on decision making: Collaborative versus individual decision making among older adults" (2007). Graduate Theses, Dissertations, and Problem Reports. 2603. https://researchrepository.wvu.edu/etd/2603

This Thesis is protected by copyright and/or related rights. It has been brought to you by the The Research Repository @ WVU with permission from the rights-holder(s). You are free to use this Thesis in any way that is permitted by the copyright and related rights legislation that applies to your use. For other uses you must obtain permission from the rights-holder(s) directly, unless additional rights are indicated by a Creative Commons license in the record and/ or on the work itself. This Thesis has been accepted for inclusion in WVU Graduate Theses, Dissertations, and Problem Reports collection by an authorized administrator of The Research Repository @ WVU. For more information, please contact researchrepository@mail.wvu.edu. 
The Effects of Framing on Decision Making: Collaborative Versus

Individual Decision Making Among Older Adults

\author{
Sarah A. Stoner
}

Thesis submitted to the Eberly College of Arts and Sciences at the West Virginia University

in Partial Fulfillment of the Requirements for the degree of

Master of Science

In

Psychology

Barry Edelstein, Ph.D., Chair

Stan Cohen, Ph.D.

JoNell Strough, Ph.D.

Department of Psychology

Morgantown, WV

2007

Keywords: Collaboration, Framing Effect, Medical Decision Making, Older Adults 


\begin{abstract}
The Effects of Framing on Decision Making: Collaborative Versus

Individual Decision Making Among Older Adults
\end{abstract}

\title{
Sarah A. Stoner
}

The framing effect in medical decision making was examined using individual and collaborative older adult decision makers. One hundred eight adults over the age of 60 participated. A lung cancer scenario was presented to each participant, with the option of choosing surgery or radiation for treatment. Participants viewed the options in either a positive (survival) or negative (mortality) frame. A mixed design was used, with frame (positive or negative) and condition (individual or collaborative) as the between subject factors, and data format (cumulative probability, interval probability, and life expectancy) as the within subject factor. Individuals demonstrated the framing effect in one data format, life expectancy, by choosing surgery in the positive frame and radiation in the negative frame more often than expected by chance.

Collaborative decision makers demonstrated the framing effect in two data formats, cumulative and interval probability. Collaborators indicated higher confidence ratings in the data format where the framing effect was not exhibited as compared to the data formats where the framing effect was exhibited. There were no other differences in confidence and use of information ratings across data formats or between decision makers who demonstrated the framing effect and decision makers who did not demonstrate the framing effect. 


\section{Acknowledgments}

There are many people I would like to thank for their roles in making the completion of this project possible. First, I would like to thank my advisor, Barry Edelstein, Ph.D., for his dedication with helping me complete this project. His guidance and support has been greatly appreciated! I would also like to extend my thanks to the other members of my committee, Stan Cohen, Ph.D. and JoNell Strough, Ph.D., for their time, feedback, and encouragement throughout this project.

I would like to express my appreciation to the members of the psychology department at Nazareth College. The guidance and training I received from them made my admission at WVU possible. I would also like to express my gratitude to the Department of Psychology Alumni Fund at West Virginia University for partially funding this project.

I would like to thank my friends in the department and my friends from home for their continued support and encouragement. Finally, I would like to express my thanks to my parents, siblings, and grandmother for their continued love, support, and encouragement. 
Table of Contents

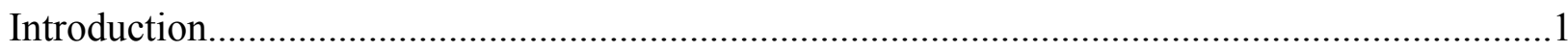

Theoretical and Conceptual Accounts of Framing...................................................2

Variability of the Framing Effect.....................................................................

Framing and Medical Decision Making...............................................................8

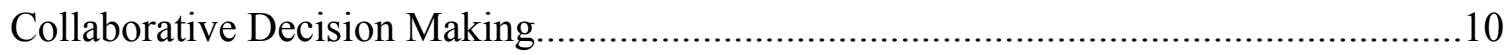

Statement of the Problem..................................................................................................... 13

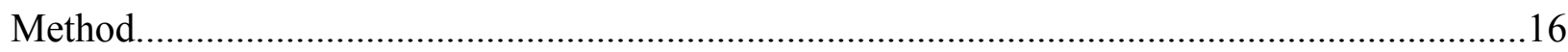

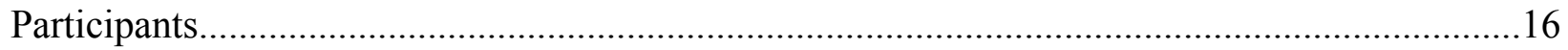

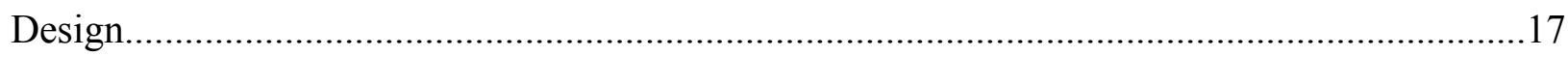

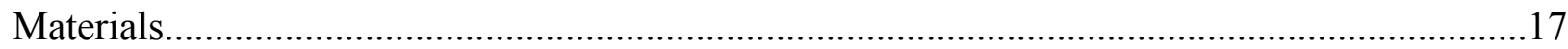

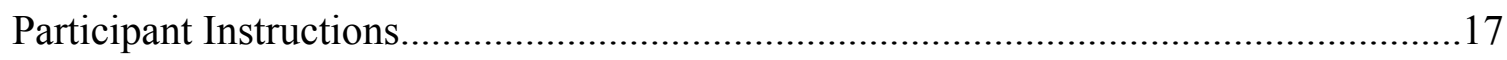

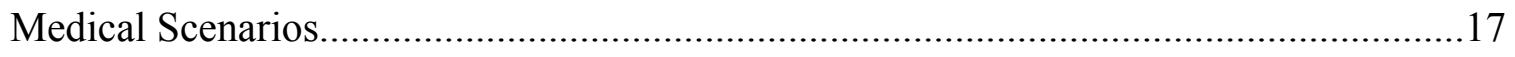

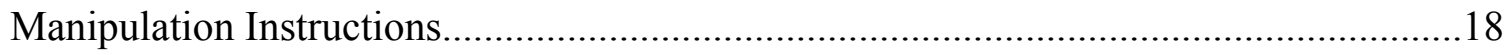

Treatment Choice Questionnaire................................................................... 18

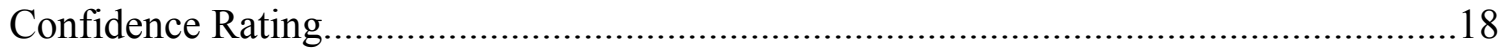

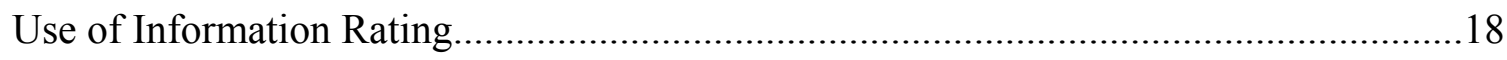

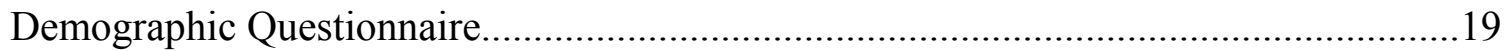

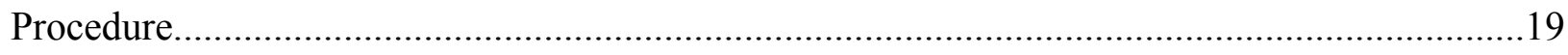

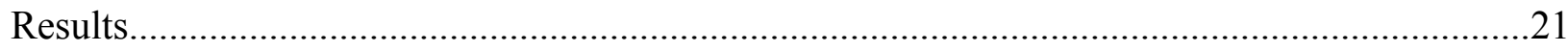

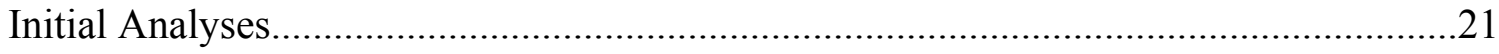

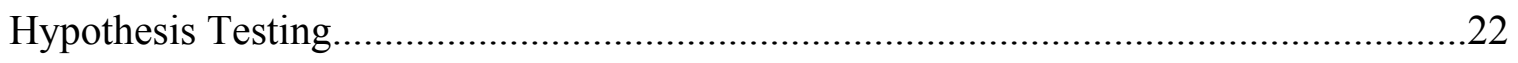

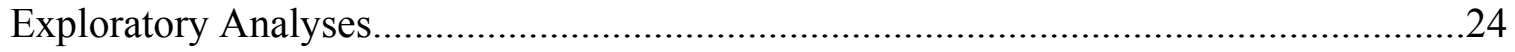


Exploratory Research Questions..........................................................................25

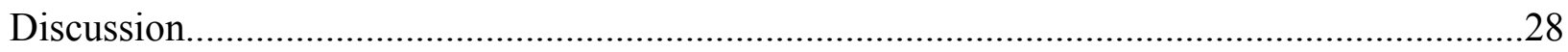

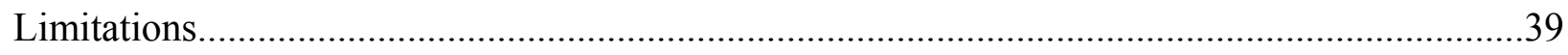

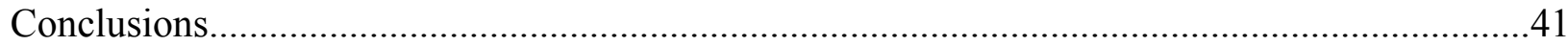

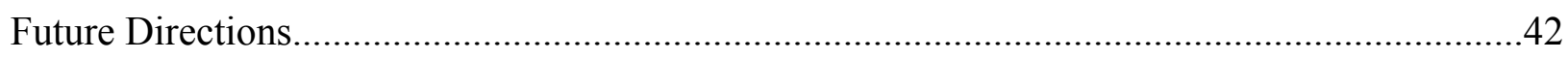

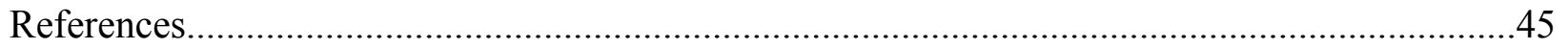

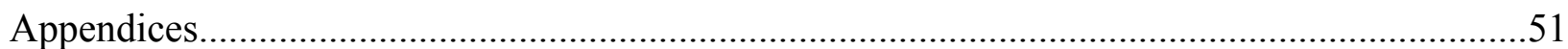




\section{List of Tables}

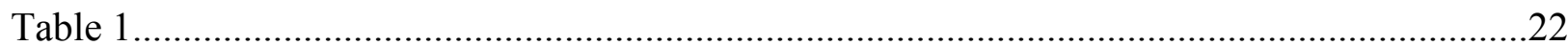

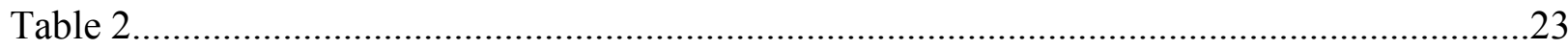

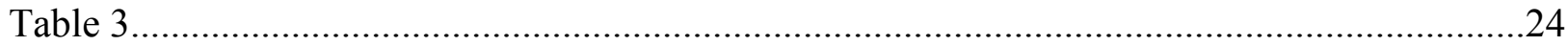


The Effects of Framing on Decision Making: Collaborative Versus Individual Decision Making Among Older Adults

The framing-effect is a well-documented bias where the manner in which options are presented influences the choices made by individuals. That is, one option is preferred when information is presented in one manner, while the other option is preferred when information is presented in a different manner. In a classic study of this effect, Tversky and Kahneman (1981) asked individuals to decide between a risky choice and a sure-outcome choice in a scenario involving a rare hypothetical disease. The question was presented in two manners, one using positive wording to describe the outcomes, and the other using negative wording:

Imagine that the U.S. is preparing for the outbreak of an unusual Asian disease, which is expected to kill 600 people. Two alternative programs to combat the disease have been proposed. Assume that the exact scientific estimate of the consequences of the programs are as follows:

Positive frame:

If Program A is adopted, 200 people will be saved.

If Program B is adopted, there is $1 / 3$ probability that 600 people will be saved, and $2 / 3$ probability that no people will be saved.

Which of the two programs would you favor?

Negative frame:

If Program C is adopted 400 people will die.

If Program $D$ is adopted there is $1 / 3$ probability that no one will die, and $2 / 3$ probability that 600 people will die.

Which of the two programs would you favor? (Tversky \& Kahneman, 1981, p. 453) 
The outcomes of options A and C are the same, as are the outcomes of options B and D. However, while option A was preferred over option B by one group of participants, option D was preferred over option $\mathrm{C}$ by a second group. By choosing option A, participants demonstrated a risk-averse preference in a positive frame; the other participant group displayed a risk-seeking preference by choosing option $\mathrm{D}$ in the negative frame. This tendency to be risk-seeking when outcomes are framed in terms of losses and risk-averse when outcomes are termed as gains has since become well documented (see Kühberger, 1998; Kühberger, Schulte-Mecklenbeck, \& Perner, 1999; Levin, Schneider, \& Gaeth, 1998; Pinon \& Gambara, 2005, for meta-analysis and reviews of the framing effect literature).

In addition to the positive and negative frames employed by Tversky and Kahneman (1981), other studies have demonstrated the framing effect by comparing gambling options presented as gains or losses (LeBoeuf \& Shafir, 2003; Takemura, 1992/1993), and medical treatment options presented in terms of the probability of survival or mortality (Armstrong, Schwartz, Fitzgerald, Putt, \& Ubel, 2002; Mashat, 2004; McKee, 2001; Woodhead, 2006). The framing effect has also been demonstrated by measuring consistency of treatment choice in medical decisions when treatment outcome is presented in different outcome formats (McNeil, Pauker, Sox, \& Tversky, 1982).

\section{Theoretical and Conceptual Accounts of Framing}

Several theories and models of decision making have been offered to better understand and explain the choices made by people when presented with differentially framed information and options. Prominent theories include utility theory (Von Neumann \& Morgenstern, 1944; Markowitz, 1952), prospect theory (Kahneman and Tversky, 1979), and cumulative prospect theory (Tversky \& Kahneman, 1992). Utility theory, based upon the assumption that time, 
computational ability, and memory abilities are limitless and flawless, states that options with the greatest expected utility are chosen when basic principles are followed during the decisionmaking process (Nelson, Stefanek, Peters, \& McCaul, 2005). That is, several basic principles (ordering alternatives, dominance, cancellation, transitivity, continuity, and invariance) allow the decision maker to weight each option according to its probability and utility, thereby determining the expected utility of the options (Markowitz, 1952; Nelson, Stefanek, Peters, \& McCaul, 2005; Von Neumann \& Morgenstern, 1947). In comparing the utilities of the alternative options, this theory assumes that individuals make rational decisions and will choose one option only if its expected utility is greater than the expected utilities of the other options (Von Neumann \& Morgenstern, 1947). However, many studies have demonstrated that individuals make decisions in violation of utility theory (i.e., Kahneman \& Tversky, 1979; see Baron, 2000 for review). Prospect theory offered an explanation for the demonstrated violations of expected utility theory, indicating that outcomes are viewed as a change from a reference point. The reference point is typically the decision maker's status quo condition, so that outcomes are perceived as either a gain or loss (Kahneman \& Tversky, 1979). Additionally, outcomes are weighted unequally according to their probability. The options with low probability are overweighted to a certain degree while options with moderate and high probability are underweighted to a stronger degree (Tversky \& Kahneman, 1981). Prospect theory and cumulative prospect theory are alike when applied to situations with two or three possible outcomes. However, cumulative prospect theory is applicable to situations with an unlimited number of outcomes. Additionally, cumulative prospect theory expands prospect theory in several ways, such as allowing the weights assigned to gains to differ from the weights assigned to losses (Tversky \& Kahneman, 1992). 
Various models of information processing have been proposed that may offer additional explanations for the presence or absence of the framing effect. For example, Yates (1990), along with Sieck and Yates (1997) suggest that decision makers often form a conclusion based solely on the information provided, rather than inferring additional information not specifically stated and incorporating this outside information into the decision making process. When presented with information framed in two different ways and requested to make a choice between options, writing or providing justification for a decision may preclude the framing effect due to an increased opportunity for decision makers to consider additional information and manipulate the given information during the decision making process (Sieck \& Yates, 1997).

Stanovich and West (2000) offer another account of information processing under framing conditions by combining similar conceptual assumptions of various dual-process reasoning theories. They suggest that information processing occurs via two routes: one is primarily holistic in nature, and the other analytic. The holistic process relies more on contextual cues and heuristics, which can be viewed as cognitive shortcuts for making faster conclusions. In contrast, the analytic process allows for depersonalizing and decontextualizing of information by incorporating more details into the decision-making process and allows individuals to disregard irrelevant information, such as the framing of the situation. In summary, many explanations of the framing effect have been offered, but there is no consensus on its cause.

\section{Variability of the Framing Effect}

Though numerous studies have demonstrated the framing effect (e.g., Armstrong, Schwartz, Fitzgerald, Putt, \& Ubel, 2002; Bernstein, Chapman, \& Elstein, 1999; Kim, Goldstein, Hasher, \& Zacks, 2005; Kühberger, 1998; Schuller, 2006), others (e.g. McElroy \& Seta, 2003; Sieck \& Yates, 1997; Takemura, 1994) have not. The framing effect has been demonstrated in 
studies where the decision concerned various topics such as health and finances (LeBoeuf \& Shafir, 2003; Kühberger, Schulte-Mecklenbeck, \& Perner, 2002; Mayhorn, Fisk, \& Whittle, 2002; Schuller, 2006), topics with little self-relevance to the participants (McElroy \& Seta, 2003), and health situations where the outcomes of hypothetical preventative surgery were presented as survival or mortality outcome curves (Armstrong et al., 2002). Additionally, the framing effect has been observed when participants were simply instructed to make a decision (Takemura, 1994), were given little time (3s or 10s) to decide (Takemura, 1992/1994), engaged primarily in holistic, automatic processing styles during the decision-making process (McElroy \& Seta, 2003), and were asked to write a justification for their decision (Levin \& Chapman, 1990). The largest effect sizes of framing have come from studies using samples of females and studies including only one risky choice option (Pinon \& Gambara, 2005). The framing effect typically yields a pattern of risk aversion in the positive frame and risk seeking in the negative frame (i.e., LeBoeuf \& Shafir, 2003; Miller \& Fagley, 1991; Tversky \& Kahneman, 1981; Takemura, 1993/1994). However, the pattern is reversed in situations involving personal medical decisions, so that the risky option is favored in the positive frame, and the nonrisky option is favored in the negative frame (i.e., McNeil, 1982; McKee, 2001; Woodhead, 2006).

The framing effect has been reduced or absent in situations where the topic was highly self-relevant to participants (McElroy \& Seta, 2003), the decision involved a hypothetical or real small monetary payoff (Kühberger, Schulte-Mecklenbeck, \& Perner, 2002), participants were allowed 3 minutes to think before indicating a decision (Takemura, 1992/1994), and participants engaged in an analytic processing style while formulating a decision (McElroy \& Seta, 2003). The framing effect also was not observed in several studies where participants were required to write a justification or rationale for their decision (Kim et al., 2005; Miller and Fagley, 1991; 
Sieck \& Yates, 1997; Takemura, 1993/1994). Mashat (2004) found some mixed results in regards to whether written justification precludes the framing effect. Woodhead (2006) found that writing precluded the framing effect in younger adults, but not older adults. In contrast to the studies where justification served to debias participants from the framing effect, Levin and Chapman (1990) found that justification did not yield a debiasing of the framing effect.

Some authors have attempted to account for the variability in the framing effect by appealing to individual difference variables. For example, this effect has been more pronounced with individuals who use holistic (reliance on contextual cues and heuristics) verses analytic (careful consideration of all presented information) thinking styles (McElroy \& Seta, 2003). Decision-making tasks that are perceived by the decision maker as highly relevant tend to induce the analytic thinking style, while decision-making tasks that are perceived by the decision maker as irrelevant tend to induce the holistic thinking style (McElroy \& Seta). Another category of individual difference variables is participant personality, which may also be predictive of susceptibility to the framing effect. For example, high ratings of conscientiousness, high ratings of neuroticism, low ratings of openness to experience, and low ratings of agreeableness have been related to demonstrating the framing effect (Levin, Gaeth, Schreiber, \& Lauriola, 2002), while high ratings of agreeableness and conscientiousness have been related to consistency in choosing risk averse options across different situations (Soane \& Chmiel, 2005). Another variable that may be related to susceptibility of framing is individual differences in need for cognition, which is the extent to which a person engages in thinking (LeBoeuf \& Shafir, 2003). Other individual difference variables that have been explored in relation to framed risky health decisions include impulsiveness, involvement in personal healthcare, and feelings towards 
personal health status, such as tendency to feel anxiety, depression, or other emotions regarding personal health status (Lauriola, Russo, Lucidi, Violani, \& Levin, 2005).

In their review of the framing literature, Levin, Schneider, and Gaeth (1998) concluded that discrepancies between results regarding the presence or absence of the framing effect may be determined by the type of framing. The authors identified three categories of framing: risky choice, attribute, and goal framing. Risky choice framing involves presentation of decision options with different risk levels. The Asian disease problem mentioned earlier is an example of a risky choice frame. Attribute framing involves manipulating the presentation of a specific characteristic of a subject. An often cited example of attribute framing comes from Levin and Gaeth's 1988 study in which meat was described as being $25 \%$ fat or as $75 \%$ lean. The positively framed information about meat ( $75 \%$ lean) was judged more desirable than the negatively framed information about meat ( $25 \%$ fat), demonstrating a framing effect for attribute manipulation. The third category of framing, goal framing, involves manipulating the framing of the outcome of a targeted behavior. In positive outcome framing, benefits obtained by performing the behavior are discussed. In negative outcome framing, the potential losses that can occur by not performing the behavior are highlighted. For example, participants in Rothman, Martino, Bedell, Detweiler, \& Salovey's (1999) study were told of the benefits of using mouthwash ("safe and effective way to reduce plaque accumulation"; p. 1361) expressed a greater intention of buying mouthwash than participants who were told of the disadvantages of not using mouthwash ("failing to take advantage of a safe and effective way to reduce plaque accumulation"; p. 1361), demonstrating a framing effect for goal framing. 


\section{Framing and Medical Decision Making}

One context in which people often need to make important, often life threatening, decisions is health. The framing effect has been demonstrated with medical scenarios in several studies (e.g., Armstrong et al., 2002; McNeil, Pauker, Soc, \& Tversky, 1982; Rothman et al., 1999). A variety of factors have been found to determine the framing effect in these kinds of scenarios. For example, Rothman et al. (1999) reported that the likelihood of performing a health detection behavior (i.e., undergoing testing for a disease) increased after young adults read about possible losses associated with not performing the detection behavior, while the likelihood of performing a preventative behavior (i.e., receiving inoculation for a disease) increased when the gains of performing the behavior were emphasized. However, individuals may be less susceptible to the framing effect when they are highly involved in the decision making process and the decision is relevant to themselves (Krishnamurthy, Carter, \& Blair, 2001). Outcome format may also be a factor that influences the framing effect. For example, McNeil et al. (1982) found that when data were presented in cumulative format (number of expected survivors/mortalities during treatment delivery, one year after treatment, and five years after treatment), radiation was preferred more than when the data were presented in life expectancy format (number of expected survivors/mortalities during treatment and number of years expected to live). McNeil et al. also found that surgery was preferred more often when stated in a survival frame as compared to a mortality frame. Additionally, Armstrong et al. (2002) found that manipulation of the presentation of possible survival/mortality outcomes following hypothetical preventative surgery (or lack of surgery) decisions made by individuals, in that more individuals opted for the preventative surgery when the survival curves were displayed than when mortality curves were displayed. 
Moxey, O'Connell, McGettigan, and Henry (2003) recently reviewed research investigating how the framing effect influences various health and medical decisions. The authors concluded that results gained from situations involving making a decision using framed information differed along with other variables, including the presented scenario, manipulation of the scenario, and individual differences among participants. For example, when individuals are presented with a positively or negatively framed scenario in which they are required to choose between surgery and some other treatment option, Moxey et al. noted that surgery is preferred more often in the positive than the negative frame. Similarly, when the choices involved invasive or toxic treatments, these options were more frequently preferred when presented in a positive frame than a negative frame. When the positively or negatively framed information pertained to health behaviors, positive frames were more effective than the negative frames for promoting these behaviors.

While many studies investigating the framing effect in healthcare decision making have used young adults as participants (Moxy et al., 2003; Pinon \& Gabara, 2005), few studies have examined the framing effect among older adults faced with health care decisions. Nevertheless, there is evidence that older adults are susceptible to the framing effect (e.g., Kim et al., 2005; Mckee, 2001). McKee demonstrated the framing effect with older adults using the cancer treatment problem used by McNeil et al. (1982). Kim et al. found the framing effect with older adults using a fatal disease problem and a cancer treatment problem similar to that used by McKee. In contrast, Woodhead (2006) presented framed cancer treatment options in three different outcome formats (cumulative probability, interval probability, life expectancy), and found different results for each format. A framing effect was present among older adults when the survival/mortality data were presented in interval probability format (number of expected 
survivors/mortalities during a specified time interval). In summary, demonstrations of the framing effect among older adults are few and inconsistent.

\section{Collaborative Decision Making}

When older adults make decisions regarding health, they often collaborate or seek advice from others (Strough, Patrick, Swenson, Cheng, \& Barnes, 2003). Collaboration has been studied in many contexts (see Meegan \& Berg, 2002 for a review), and may be beneficial with some tasks that employ similar skills needed for decision making. For example, Saczynski, Margrett, and Willis (2004) found that when trained individually or as part of a collaborative dyad, older adults learned problem solving strategies for completing tasks involving inductive reasoning skills to the same extent. However, collaborators maintained the problem-solving strategies to a greater degree than individuals, as evidenced by better performance on problems solved collaboratively at a 3-month posttest. Dixon and Gould (1998), and Gould, Trevithick, and Dixon (1991) found that older adults recalled more information from narratives and provided more elaborations when collaborating in tetrads as compared to collaborating in dyads or performing individually.

In contrast to studies indicating that collaboration may be associated with some benefits, some literature suggests that collaborators do not perform as well as nominal pairs (scores based on combined individual scores) on some tasks. Andersson and Rönnberg (1997) created nominal pairs by combining the scores obtained by two individuals completing a recall task alone. For example, one individual may correctly recall items A, B, and C on a given task, for a total of three recalled items. A second individual may recall items B, D, and E, for a total of three recalled items. Summed together, the predicted correct recall between the two individuals would be A, B, C, D, and E, for a total of 5 recalled items. Therefore, if the two individuals were to 
engage in the task together, a score of 5 recalled items would be predicted. While dyads could recall more words than individuals, dyads failed to recall as many words as would be predicted based on nominal pair performances. Similar results have been found in other studies (Johansson, Andersson, \& Rönnberg, 2000/2005). These studies indicate that collaboration between dyads may lead to better memory performance when compared to one individual's score, but collaboration does not necessarily yield the better results than nominal pairs.

Decision-making biases have been found in group decisions. For example, the sunk-cost bias occurs when individuals continue to pursue a failing plan of action in which they've previously invested a significant amount of resources, rather than change the course of action and pursue a more economically rational option (Arkes \& Blumer, 1985). Though individuals and groups evidence this sunk-cost bias (Smith, Tindale, \& Steiner, 1998), groups may show a stronger severity of this bias than individuals (Whyte, 1993). Additionally, when making group decisions, individuals within the group show a confirmation bias, wherein they focus on gathering confirmatory rather than disconfirmatory information when the majority of group members share a preference for a particular choice (Schulz-Hardt, Frey, Lüthgens, \& Moscovici, 2000).

Studies examining the susceptibility of dyads to the framing effect bias are lacking. However, one study examined the framing effect in group decisions involving three individuals (Paese, Bieser, \& Tubbs, 1993). Participants were initially presented with either four positively framed scenarios or four negatively framed scenarios, and asked to decide between risky and non-risky options. The scenarios involved the Asian disease problem, a financial problem for a hypothetical company, a medical scenario involving making a choice to receive surgery for a heart problem, and a money gambling problem. Within each of the scenarios, participants were 
presented with nine sets of decisions. Choice A for each of the 9 decisions was a sure option and did not vary among the nine items. Choice B for each of the 9 decisions was a risky option, and the probability of the desired outcome increased from $10 \%$ on item 1 to $90 \%$ on item 9 . Therefore, on each of the four scenarios, individuals had to decide between risky options and non-risky options nine times. Groups were then formed using the same participants. Some groups were composed of individuals who had all been exposed to the positively framed scenarios. Half of these groups were presented with the positively framed information again, while the other half was given the negatively framed scenarios. The other groups were composed of individuals who had been exposed to the negatively framed information. Again, half of these groups were given the positively framed scenarios, while the other half received the negative scenarios. Each group reported a group response for each of the 9 items in all four scenarios. Among the decisions made individually, the framing effect was exhibited across the four scenarios. However, group results differed for each of the four scenarios. In the Asian disease scenario, all groups reported more risk aversion than was reported individually, and there was a main effect for frame so that groups presented with the positive frame were more risk averse than groups presented with the negative frame. When presented with the financial decision, the main effect of frame was present so that groups presented with the positive frame were more risk averse than groups presented with the negative frame. There was also an interaction between frame and the individual/group condition with the financial decision, so that the framing effect was weakened when the participants received one framed version individually (positive or negative) and the other framed version in the group (positive or negative). In the medical scenario, groups exhibited more risk-aversion across framing conditions (positive or 
negative) compared to choices made individually. In the money gambling scenario, group decisions were generally more risk-taking than individual decisions.

The collaborative decision making and problem solving literature does not afford us with evidence to draw conclusions regarding the susceptibility of collaborators to decision-making biases and their consequences, such as the framing effect. What appear to be the only related literature has focused on the effects of biases on larger group decision-making (i.e., Arkes \& Blumer, 1985; Paese et al., 1993; Smith et al., 1998).

In addition to the paucity of collaborative decision-making research, investigators have failed to include older adults as participants. Therefore, previous results may not be generalizable to this age group.

\section{Statement of the Problem}

The framing effect has been found in a variety of different contexts and situations (e.g., Armstrong et al., 2002; Bernstein et al., 1999; Tversky \& Kahneman, 1981). These have included studies of the effects of framing on older adult medical decisions (e.g., Kim et al., 2005; McKee, 2001; Woodhead, 2006). Several theories and models, including utility theory (Markowitz, 1952; Von Neumann \& Morgenstern, 1944), prospect theory (Kahneman and Tversky, 1979), cumulative prospect theory (Tversky \& Kahneman, 1992), and models of information processing (i.e., Sieck \& Yates, 1997; Stanovich \& West, 2000; Yates, 1990), have offered explanations for the framing effect. However, there is no consensus among them as to why the framing effect occurs and how it can be prevented.

Some previous studies have explored methods through which the framing effect can be precluded or minimized (e.g., McElroy \& Seta, 2003; Sieck \& Yates, 1997; Takemura, 1994). Some strategies have been successful in enabling individuals, including older adults, to avoid the 
framing effect, although none of them appear to be effective with everyone. Differences in the influence of framing might be explained through examining individual differences of the decision makers (e.g., LeBoeuf \& Shafir, 2003; McElroy \& Seta, 2003; Soane \& Chmiel, 2005) or by examining the different types of framing (risky choice, attribute, goal; Levin et al., 1998), yet the research is not conclusive.

Collaboration among individuals making medical decisions has largely been ignored in the literature even though older adults commonly collaborate with others in making medical decisions (cf., Strough et al, 2003). There is a modest amount of research supporting the advantages of collaboration on memory tasks (e.g., Dixon \& Gould, 1998; Gould, Trevithick, \& Dixon, 1991; Saczynski, Margrett, \& Willis, 2004). However, there is evidence that collaboration is not always better than individual performance (e.g., Andersson \& Rönnberg, 1997). The effects of collaboration on medical decision making in general and on the susceptibility to framing effects in particular remain uninvestigated. It is possible that collaboration precludes a framing effect. For example, collaborative decision making could naturally encourage discussion that is consistent with analytic approaches to decision making, and there is evidence that methods encouraging more analytic consideration of decision rationales may preclude the framing effect with individuals (McElroy \& Seta, 2003). However, collaboration does not always yield results predicted by individual performance, nor have groups overcome other decision-making biases. Indeed, groups of decision makers have exhibited the confirmation bias (Schulz-Hardt et al., 2000). If the collaborators in this study initially agree on a treatment option, they may not consider disconfirmatory information during their discussion of treatment options. Therefore, it could be that older adults still exhibit the framing effect after 
collaborating, and that other methods for precluding the framing effect are necessary for individuals faced with medical decisions.

The purpose of the present study was to investigate medical decision making among older adults faced with positively or negatively framed information regarding a choice between receiving surgery or radiation for treating lung cancer. This study addressed four questions. The first question was whether individual decision makers would exhibit the framing effect in at least one of three data formats (cumulative probability, interval probability, total life expectancy). Based on findings of previous studies (i.e., Armstrong et al., 2002; Kim et al., 2005; McKee, 2001; McNeil et al., 1982; Woodhead, 2006), the hypothesis for this question was that the proportion of individuals choosing surgery in the survival frame would be significantly different from the proportion of individuals choosing surgery in the mortality frame in at least one data format, indicating that the framing effect was present.

The second question was whether decision makers who made a decision as part of a dyad would demonstrate the framing effect. No hypothesis was offered for this question in light of insufficient relevant theoretical and empirical work.

If a framing effect was exhibited in one of the two conditions (individual decision making or dyad decision making), the third question asked if the proportion of decision makers exhibiting the framing effect would differ between the individual and group conditions. Again, no hypothesis was offered for the foregoing reasons.

The fourth question was to what extent the following variables contribute to the prediction of the medical decision: confidence in having made the best decision, extent to which the decision was based on the presented information, sex, experience with cancer and cancer treatment, individually vs. group decision making, or frame (survival vs. mortality)? This 
question was intended to partially replicate the findings of Woodhead (2006) with regard to predictors of treatment choice, as well as extend her findings to individual versus group and confidence in decisions. Frame (survival vs. mortality) was hypothesized to be a significant predictor based on previous research (e.g., McKee, 2001; Woodhead, 2006). Surgery was hypothesized to be more likely to be preferred in the survival frame and radiation was hypothesized to be more likely to be preferred in the mortality frame. The remaining variables (confidence in having made the best decision, extent to which the decision was based on the presented information, sex, experience with cancer and cancer treatment, individually vs. group decision making) were exploratory, as previous literature has not explored them in this context. Method

\section{Participants}

One hundred eight older adults (aged 60 years and older) were recruited from 14 senior citizen centers, one assisted living facility, and one health fair at a local mall over the course of 9 weeks. Inclusion criteria included the ability to read the materials presented in the study and speak English. The sample size was calculated using a power analysis based on findings of a previous study (Woodhead, 2006). The sample size was estimated to yield a power of at least 0.70 for a majority of the planned analyses.

Each potential participant was asked to identify someone who was present at the recruitment site with whom they would consult when they had to make a decision. If both people agreed to participate, they were randomly assigned to work individually or work together. Occasionally, a potential participant could not identify someone who was present at the site with whom they would discuss an important decision. These participants were then assigned to the individual condition. Thirty six older adults were assigned to complete the study materials 
independently. The other 72 participants were divided into 36 dyads. One participant in each dyad was randomly designated the decision maker, and the other participant in each dyad was designated the non-decision maker. Dyads consisted of married couples, relatives, or friends. Design

This study used a mixed design with two between subject variables (frame and condition) and one within subject variable (data format). The frame variable had two levels: positive/survival wording and negative/mortality wording. The condition variable also had two levels: individual and dyad. Data format was the within-subject variable, which had three levels: cumulative probability, interval probability, and total life expectancy. The study included six dependent variables: treatment choice, confidence rating, use of information rating, experience with cancer and cancer treatment, and sex. Analyses included the choices and ratings indicated by the individuals, and the choices and ratings indicated by the collaborative decision maker. Information gathered from collaborative non-decision makers was not analyzed in this study.

\section{Materials}

Participant Instructions. Participants received instructions (see Appendix A) providing background information for lung cancer, treatment choices (surgery vs. radiation) that were offered in the scenarios, and information stating the level of expected recovery 6-weeks after the procedures. The instructions asked participants to complete the questions in order without returning to any previous page.

Medical Scenarios. Three medical scenarios, previously used in Woodhead (2006) and Mashat (2004), served as the stimulus materials by providing participants with information regarding lung cancer treatment options (see Appendix B). Each scenario presented information regarding the outcome of surgery and radiation procedures in cumulative probability, interval 
probability, or total life expectancy format. The information was presented in either a positive frame (survival wording), or a negative frame (mortality wording). The wording of frames was different but the outcomes were the same. Therefore, six scenarios were used: cumulative probability: survival, cumulative probability: mortality, interval probability: survival, interval probability: mortality, total life expectancy: survival, and total life expectancy: mortality.

Manipulation instructions. Of the participants assigned to the dyad condition, one person was designated as the decision maker. The decision maker was instructed to engage in a conversation with his or her partner regarding the information presented before indicating his or her decision. The dyad was instructed to discuss the decision as long as necessary before making a decision. Participants assigned to the individual condition were instructed to think about the decision as long as necessary before making a decision (see Appendix C). The appropriate manipulation instructions appeared on the same page as the medical scenarios in the participants' packets.

Treatment choice questionnaire. Individual decision makers and collaborative decision makers were given a form on which to indicate their preferred method of treatment (surgery or radiation; see Appendix D). The participants identified as the non-decision makers in the dyad condition did not receive this questionnaire.

Confidence rating. Following the statement of treatment preference, decision makers were asked to assign a rating of 1 to 10 , indicating their confidence that they made the best decision (see Appendix E). The non-decision maker in each dyad was asked to complete a similar form (see Appendix F).

Use of information rating. Participants were asked to perform two ratings (see Appendix G) regarding the use of the information presented. First, they were asked to assign a rating on a 
10 point Likert-type scale that indicated the extent to which they based their decision on the information presented. They were then asked to perform another rating on another 10 point Likert-type scale assessing how thoroughly they considered the information. The non-decision maker in each dyad was asked to complete similar ratings (see Appendix $\mathrm{H}$ ).

Demographic questionnaire. A demographic questionnaire (see Appendix I) was distributed to each participant. The questionnaire was similar to those used by Woodhead (2006), Mashat (2004), and McKee (2001). Questions on the demographic questionnaire included age, sex, ethnicity (race), marital status, years of marriage, years of education, subjective health rating, and experiences with cancer. A four-item questionnaire from the Multilevel Assessment Instrument (Lawton, Moss, Fulcomer, \& Kleban, 1982) was included in order to determine the participant's perceived health status.

\section{Procedure}

Participants were assigned to one of two conditions: individual or dyad. Each dyad consisted of an individual and a friend. One member of the dyad was designated as the decision maker, with the assumption this person would ultimately receive the hypothetical treatment. Therefore, that person was instructed to indicate his or her final choice.

Participants were given a consent form and provided an opportunity to ask questions. Informed consent was obtained before continuing. All participants were given a questionnaire packet that contained either positively or negatively framed scenarios. Page one was the participant instructions, which included information on lung cancer treatment. Page two contained the information in one of the data formats in either a positive or negative frame and either the individual, dyad decision maker, or dyad non-decision maker manipulation instructions. The treatment choice questionnaire was presented on page three. The presentation 
order of one data format, manipulation instructions, and treatment choice questionnaire were repeated on the following pages for the remaining two data formats, so that all packets contained all three data formats. Following the page with the treatment choice questionnaire for the third data format, participants received confidence rating scales and use of information scales. They completed these scales for each of the three data formats. These scales were presented at the end in order to prevent them from serving as debiasing mechanisms. Within the packets, the scenarios were presented in six different orders in order to control for order effects: 1) cumulative probabilities (C), interval probabilities (I), life expectancy (L), 2) C, L, I, 3) I, L, C, 4) I, C, L, 5) L, C, I, and 6) L, I, C. Twelve participants completed the questionnaire in each order.

Participants who were in the dyad condition but were not the designated decision maker received the same packet as decision makers minus the treatment choice questionnaires. When the dyad completed the decisions for each data format, the non-decision maker completed confidence rating forms and use of information forms independently. Participants in the dyad condition then completed a perceptions questionnaire, and all participants completed a future time perspective questionnaire and a modified Asian disease scenario. The data from the perceptions questionnaire, future time perspective questionnaire, and modified Asian disease problem were not analyzed in this study. The final pages of the testing packet consisted of a demographic questionnaire. Participants completed the questionnaire packets in less than one hour. Deliberations between the dyads were not timed, but most ranged from under one minute to approximately five minutes. Upon completion of the questionnaire, participants were then given the opportunity to enter a raffle for a prize of $\$ 100$ and thanked for their participation. 
Results

\section{Initial Analysis}

The individual decision maker and collaborative decision maker groups were compared on demographic variables before conducting analyses to verify that the groups did not differ. The mean age of the individual decision makers $(M=76.94, S D=9.40)$ did not differ significantly from the mean age of the collaborative decision makers $(M=76.47, S D=7.44$; $t(70)=.236, p=.814)$. Additionally, the average number of years of education did not significantly differ between the individual decision makers $(M=12.03, S D=2.47)$ and the collaborative decision makers $(M=12.66, S D=2.47 ; t(65)=-1.039, p=.302)$. One participant in the individual condition was African American, the rest reported their ethnicity as Caucasion. All participants in the collaborative decision maker condition reported their ethnicity as Caucasion. Both groups were predominantly female; $75 \%$ of the individual decision makers $(N$ $=27)$ and $86.1 \%$ of the collaborative decision makers $(\mathrm{N}=31)$ were female. Eight individual and 12 collaborative decision makers had been diagnosed with cancer at some point during their lifetime, while 28 individual decision makers and 24 collaborative decision makers stated that they knew someone who'd been diagnosed with cancer. Twenty one of the individual decision makers stated that they'd discussed the experience of having cancer with someone who had cancer, and 19 individual decision makers stated that they had discussed cancer treatment with someone who had cancer. Of the collaborative decision makers, 22 participants stated that they'd discussed the experience of having cancer with someone who had cancer, and 21 participants stated that they had discussed cancer treatment with someone who had cancer. 


\section{Hypothesis Testing}

The first hypothesis was that the framing effect would be apparent in the decisions chosen by individual decision makers in at least one of the three data formats. A framing effect would be present if significantly more participants than expected chose surgery in the survival format and radiation in the mortality format. The percentage of collaborative decision makers who chose surgery or radiation in the survival or mortality frame for each data format is presented in Table 1. A 2x2 (frame by condition) chi-square analysis was conducted for each data format, so that three chi-square analyses were conducted. The Pearson chi-square statistic was reported.

Table 1

Treatment Choices and Significance for Individual Decision Makers

\begin{tabular}{lllllll}
\hline & \multicolumn{2}{l}{$\begin{array}{l}\text { Cumulative Format } \\
(\mathrm{n}=36)\end{array}$} & \multicolumn{2}{l}{$\begin{array}{l}\text { Interval Format } \\
(\mathrm{n}=36)\end{array}$} & \multicolumn{2}{l}{$\begin{array}{l}\text { Life Expectancy Format* } \\
(\mathrm{n}=36)\end{array}$} \\
\hline Frame & Surgery & Radiation & Surgery & Radiation & Surgery & Radiation \\
\hline Survival & 9 & 9 & 11 & 7 & 12 & 6 \\
\hline Mortality & 5 & 13 & 7 & 11 & 6 & 12 \\
\hline${ }^{*} p<0.05$ & & & & & &
\end{tabular}

A chi-square analysis revealed that the percentages of participants who chose surgery in the survival frame, and radiation in the mortality frame, did not significantly differ when participants responded to the cumulative format $\left[\chi^{2}(1, N=36)=1.870 ; p=.171\right]$ or interval format $\left[\chi^{2}(1, N=36)=1.778 ; p=.182\right]$. The distribution of responses in the interval format reflect the pattern of choices exhibited when the framing effect occurs, yet the change in the distribution of scores across frames is not large enough to reach significance. However, the percentage of participants who chose surgery in the survival frame, and radiation in the mortality frame, was significantly different than expected by chance in the life expectancy format $\left[\chi^{2}(1, N\right.$ 
$=36)=4.0 ; p=.046]$. That is, more people than expected by chance chose surgery instead of radiation when presented with the positively framed information, and more people than expected by chance chose radiation instead of surgery when presented with the negatively framed information. This finding indicates that the framing effect occurred in the life expectancy format, but not in the cumulative or interval format.

The second hypothesis tested whether the participants who made decisions as part of a dyad would demonstrate the framing effect. The percentage of collaborative decision makers who chose surgery or radiation in the survival or mortality frame for each data format are presented in Table 2. A 2x2 (frame by condition) chi-square analysis was conducted for each data format, so that three chi-square analyses were conducted. The Pearson chi-square statistic was reported.

Table 2

Treatment Choices and Significance for Collaborative Decision Makers

\begin{tabular}{lllllll}
\hline & \multicolumn{2}{l}{$\begin{array}{l}\text { Cumulative Format* } \\
(\mathrm{n}=36)\end{array}$} & $\begin{array}{l}\text { Interval Format* } \\
(\mathrm{n}=36)\end{array}$ & \multicolumn{3}{l}{$\begin{array}{l}\text { Life Expectancy Format } \\
(\mathrm{n}=36)\end{array}$} \\
\hline Frame & Surgery & Radiation & Surgery & Radiation & Surgery & Radiation \\
\hline Survival & 13 & 5 & 12 & 6 & 11 & 7 \\
\hline Mortality & 6 & 12 & 5 & 13 & 6 & 12 \\
\hline \multicolumn{2}{c}{$* 0.05$} & & & & &
\end{tabular}

A chi-square analysis revealed that the percentage of collaborative decision makers who chose surgery in the survival frame and radiation in the mortality frame was significantly different than expected by chance in the cumulative format $\left[\chi^{2}(1, N=36)=5.461 ; p=.019\right]$ and the interval format $\left[\chi^{2}(1, N=36)=5.461 ; p=.019\right]$. In both of these formats, a larger proportion of people than expected by chance chose surgery instead of radiation in the survival frame, and a larger proportion of people than expected by chance chose radiation instead of 
surgery in the mortality frame. However, the difference between expected and observed choices was not significant in the life expectancy format $\left[\chi^{2}(1, N=36)=2.786 ; p=.095\right]$. The distribution of responses in the life expectancy format reflect the pattern of choices exhibited when the framing effect occurs, yet the change in the distribution of scores across frames is not large enough to reach significance. These results indicate that collaborative decision makers exhibited the framing effect when presented with data in the interval and cumulative formats, but not in the life expectancy format.

Exploratory Analyses

The third research question asked if the proportion of decision makers exhibiting the framing effect differed between the individual and collaborative conditions. Participant's treatment choices were coded as exhibiting the framing effect if the participant had a choice reversal for any of the three data formats. Participants whose choices were consistent across data formats were coded as not exhibiting the framing effect. This coding technique is consistent with coding used in Woodhead (2006). A chi-square analysis was conducted to determine if the proportion of participants exhibiting the framing effect differed more than expected between the individual and collaborative condition. Results of these analyses are presented in Table 3. Table 3

Number of Decision Makers Exhibiting the Framing Effect

\begin{tabular}{lll}
\hline Condition & Did Exhibit & Did Not Exhibit \\
\hline Individual & 12 & 24 \\
\hline Dyads & 6 & 30 \\
\hline \multicolumn{1}{c}{$* 0.05$} & &
\end{tabular}

The results revealed that the proportion of participants who exhibited the framing effect in the individual or collaborative condition did not differ significantly more than expected, $\left[\chi^{2}(1\right.$, 
$N=72)=2.667 ; p=.102]$. Neither group showed a significantly stronger susceptibility to the framing effect.

The fourth question was an exploratory question, which examined the extent to which susceptibility to the framing effect could be predicted by the following variables: making the decision individually or collaboratively, confidence in decision, extent to which the decision maker reported using the information presented in the scenarios, gender, or experience with cancer and cancer treatment. Experience with cancer and cancer treatment included having been diagnosed with cancer, having someone close diagnosed with cancer, having discussed the experience of having cancer with someone, or having discussed the treatment of cancer with someone. This hypothesis was addressed using a logistic regression analysis where exhibiting the framing effect was the criterion variable. The overall model was not significant $\left(\chi^{2}(5, N=61)=\right.$ $3.261 ; p=.66)$. Furthermore, none of the variables accounted for a significant amount of variance in explaining which people exhibited the framing effect.

Exploratory Research Questions

Four research questions were addressed. The first and second exploratory research questions were within-subject comparisons of confidence and use of information ratings between data formats where the framing effect was exhibited and data formats where the framing effect was not exhibited. Neither the individual nor collaborative decision makers exhibited the framing effect in all three data formats, thereby making this comparison possible. The third and fourth exploratory research questions were between-subject comparisons of confidence and use of information ratings between decision makers who exhibited the framing effect and decision makers who did not exhibit the framing effect. 
The first exploratory research question asked if confidence ratings differed between data formats where the framing effect occurred and data formats where the framing effect did not occur. Individual decision makers' and collaborative decision makers' ratings were addressed separately because they exhibited the framing effect in different data formats.

The individuals demonstrated the framing effect in the life expectancy format, and did not demonstrate the framing effect in the cumulative and interval formats. There was not a significant difference in confidence ratings in the cumulative format $(\mathrm{M}=20.67, S D=6.75)$ and the interval format $(\mathrm{M}=20.47, S D=6.88 ; t(29)=.23, p=0.82)$ so the confidence ratings were averaged across these two formats. A comparison of the confidence scores given by individuals in the format where they did exhibited the framing effect (life expectancy; $\mathrm{M}=20.13, S D=$ $5.90)$ and the formats where they did not exhibit the framing effect $(\mathrm{M}=20.57, S D=6.38)$ revealed no significant difference $(t(29)=-0.613, p=0.54)$.

The collaborative decision makers demonstrated the framing effect in the cumulative and interval formats, but not in the life expectancy format. The confidence ratings in the cumulative format $(\mathrm{M}=21.63, S D=5.16)$ did not differ significantly from the confidence ratings in the interval format $(\mathrm{M}=21.25, S D=4.70 ; t(31)=0.671, p=0.51)$, so the data was averaged across these two conditions. A comparison of confidence scores given by collaborative decision makers in the formats where the framing effect was demonstrated $(\mathrm{M}=21.44, S D=4.68)$ and the format in which it wasn't demonstrated (life expectancy, $\mathrm{M}=22.75, S D=5.07$ ) revealed a significant difference $(t(31)=2.09, p=0.045)$. Collaborative decision makers were more confident in their decisions in the format where the framing effect did not occur than in the format where the framing effect did occur. 
These analyses indicate that individual decision makers felt equally confident in their decisions in data formats where they were and were not demonstrating the framing effect. However, the collaborative decision makers rated their confidence lower in data formats where they demonstrated the framing effect.

The second exploratory research question asked if the use of information ratings differed between the formats in which the framing effect did and did not occur. Again, ratings by individual decision makers were analyzed separately from ratings by collaborative decision makers. Among the individual decision makers, there was no significant difference between the use of information scores reported in the two data formats in which they did not demonstrate the framing effect (cumulative format: $M=13.80, S D=4.93$; interval format: $M=14.00, S D=$ $5.79 ; t(29)=-0.32, p=0.75)$. The data was averaged across these two conditions. Use of information ratings did not significantly differ between the format in which the framing effect occurred $(M=13.87, S D=5.59)$ and the formats in which the framing effect did not occur $(M=$ $13.90, S D=5.10 ; t(29)=-0.03, p=0.97)$. Among the collaborative decision makers, there was no significant difference in use of information ratings in the data formats where they demonstrated the framing effect (cumulative format: $M=15.67, S D=4.71$; interval format: $M=$ $15.18, S D=5.15 ; t(32)=0.889, p=0.381)$, so data were averaged across these conditions. Use of information scores did not significantly differ between the formats in which the framing effect occurred $(M=15.42, S D=4.68)$ and format in which the framing effect did not occur $(\mathrm{M}=$ $15.79, S D=4.72 ; t(32)=1.02, p=0.31)$. These analyses showed that neither individual nor collaborative decision makers reported differences in the way they used the information presented in the data formats where the framing effect was demonstrated as compared to data formats where the framing effect wasn't demonstrated. 
The third exploratory research question asked if people who demonstrated the framing effect differed in their confidence ratings from those who did not demonstrate the framing effect. The total confidence in choice across the three data formats did not differ between decision makers who exhibited the framing effect $(N=18, M=60.0, S D=12.86)$ and decision makers who did not exhibit the framing effect $(\mathrm{N}=54, \mathrm{M}=64.84, S D=16.71 ; t(60)=1.08, p=0.29)$. This indicates that individuals were similarly confident in their decisions regardless of whether they exhibited the framing effect.

The final exploratory research question asked if the decision makers who demonstrated the framing effect and the decision makers who did not demonstrate the framing effect differed in their reported use of information. The extent to which people rated using the information presented in making their decision did not differ between individuals who demonstrated the framing effect $(N=18, M=43, S D=10.94)$ and those who did not demonstrate the framing effect $(N=54, M=44.74, S D=14.94 ; t(61)=0.438, p=0.66)$. This indicates that individuals who demonstrated the framing effect did not differ in their use of information from individuals who did not demonstrate to the framing effect.

\section{Discussion}

This study posed four research questions with two hypotheses; both hypotheses were supported. Older adult individual decision makers and older adult collaborative decision makers demonstrated the framing effect in at least one of the three data formats presented. There was no difference between the proportion of individual and collaborative decision makers who demonstrated the framing effect. Furthermore, the framing effect was not predicted by gender, previous experience with cancer and cancer treatment, confidence ratings, use of information ratings, or having made the treatment decision individually or collaboratively. The findings 
discussed in further detail for each research question. Results from four exploratory analyses are also discussed.

Framing effect among individual decision makers

Older adults who completed the decision making task individually demonstrated the framing effect in one data format: life expectancy. In this format, older adults who made their decision without input from others demonstrated the framing effect by choosing surgery in the positive frame and radiation in the negative frame more often than expected. This pattern of preferring surgery in positive frames (risk seeking) and radiation in negative frames (risk avoidance) is consistent with previous studies. McNeil et al. (1982) demonstrated this pattern with students, physicians, and patients, while LeBoeuf and Shafir (2003) found this pattern with undergraduates. McKee (2001) and Woodhead (2006) demonstrated this pattern with older and younger adults. However, this pattern of risk-seeking in the positive frame and risk-avoidance in the negative frame is opposite to findings obtained using the Asian disease problem (LeBoeuf \& Shafir, 2003; Miller \& Fagley, 1991; Takemura, 1994; Tversky \& Kahneman, 1981), a money problem in which a choice between a risk-free outcome of $\$ 400$ or an risky outcome of either $\$ 300$ or $\$ 500$ was perceived as a gain or a loss from an initial amount of money (LeBoeuf \& Shafir, 2003), and similar money problems involving different amounts of money (Takemura, 1992/1993). The pattern, and therefore the support for different decision making theories, varies according to the type of scenario presented.

The documentation of the framing effect in only the life expectancy format with the individual decision makers is consistent with one previous study (McKee, 2001), but inconsistent with other previous studies (Woodhead, 2006; McNeil et al., 1982) The framing effect was demonstrated in the life expectancy format in a within-subject study of older adult participants 
(McKee). In contrast, a sample of patients, physicians, and students demonstrated the framing effect in the cumulative probability and life expectancy formats (McNeil et al.). However, with a sample of older adults, Woodhead (2006) demonstrated the framing effect in the interval probability format, but not in the cumulative probability or life expectancy formats. LeBoeuf and Shafir (2003) demonstrated the framing effect among college students using the lung cancer scenario, but did not indicate which data formats were tested. The inconclusive evidence regarding which data formats consistently yield the framing effect is compounded by the variations in the designs of studies examining the framing effect (i.e., within-subject, betweensubject). Based on these previous inconsistencies in documenting the framing effect using three lung cancer scenarios, it is unclear why the individual decision makers in this study demonstrated the framing effect in the life expectancy format, and not the other formats. Framing effect among collaborative decision makers

The collaborative decision makers demonstrated the framing effect in both the cumulative and interval probability formats. That is, older adults who collaborated with a peer prior to indicating their preference for surgery or radiation demonstrated the framing effect by choosing surgery in the positive frame and radiation in the negative frame more often than would be expected. However, the framing effect was not demonstrated in the life expectancy format. From the information gathered in this study, it is unclear why the individual and collaborative decision makers failed to demonstrate the framing effect in any of the same data formats.

The findings for the collaborative decision makers are not directly comparable to previous research, as no studies have examined the framing effect among older adult collaborative decision makers. However, in the current study, the individual and collaborative decision makers did not demonstrate the framing effect in the same data formats. This finding is 
interesting because it could suggest that there were some differences between the individual and collaborative decision making processes. These differences could be attributed to the collaborative decision makers' opportunity to discuss the options with another person, and their possible gain of additional information through this process. In contrast, the individual decision makers had no similar opportunity for discussion. We were hopeful that the collaborators' opportunity for discussion would preclude the framing effect; however, the collaborative decision makers exhibited the framing effect in more formats than the individual decision makers. A comparison of the collaborative decision makers' choices with individual decision makers' choices from previous studies involving the lung cancer scenario (LeBoeuf \& Shafir, 2003; McKee 2001; McNeil, 1982; Woodhead, 2006) is difficult. While the previous studies demonstrated the framing effect using a variety of population samples (students, physicians, patients, older adults), the framing effect was not consistently demonstrated in any one data format. Based on the previous inconsistencies in documenting the framing effect using the lung cancer scenarios, it is unclear why the collaborative decision makers in this study demonstrated the framing effect in the cumulative and interval probability format, and not the life expectancy format. One possibility is that the vignettes used in these studies are unreliable in producing the framing effect. On the other hand, not all individuals exhibit the framing effect, and previous sample sizes may have been insufficient for providing enough power to detect the framing effect. Collaborative decision making was a unique element of this study, as the framing effect has not been previously examined among collaborative decision makers. It is not uncommon for older adults to collaborate or seek advice from others when making health-related decisions (Strough, Patrick, Swenson, Cheng, \& Barnes, 2003). The framing effect was demonstrated with the collaborative decision makers, indicating that older adults are influenced by framed 
information even when provided with the opportunity to collaborate with another person. While this is the first study documenting the framing effect with older adult dyads, other decision making biases have been exhibited by collaborating groups of decision makers (i.e., confirmation bias, Schultz-Hardt, Frey, Lüthgens, \& Moscovici, 2000; sunk-cost bias, Smith, Tindale, \& Steiner, 1998; Whyte, 1993). The evidence from the current study and previous studies (SchultzHardt et al,; Smith, Tindale, \& Steiner; Whyte) suggest that collaborating with others is not sufficient for precluding decisional biases.

\section{Application to decision making theories}

The pattern of preferring the risky choice, surgery, in positive frames and the non-risky choice, radiation, in negative frames is consistent with previous studies examining the framing effect in individual medical treatment choice (McNeil, 1982; McKee, 2001; Woodhead, 2006). However, this pattern is a reversal of the pattern evidenced in the other studies using the Asian disease problem (LeBoeuf \& Shafir, 2003; Miller \& Fagley, 1991; Tversky \& Kahneman, 1981), or problems involving money (LeBoeuf \& Shafir, 2003; Takemura, 1993/1994). In these studies, the non-risky choices were preferred in positive frames, while risky choices were preferred in negative frames. This reversal in risk-seeking has not yet been addressed in the studies demonstrating the change.

The present findings are inconsistent with the predictions of utility theory (Von Neumann \& Morgenstern, 1944; Markowitz, 1952). Had the decisions in this study been consistent with the decisions predicted by utility theory, preference for surgery or radiation would have been equivalent across frames. However, decisions are often made in violation of utility theory (Baron, 2000). 
The findings in this study and previous similar studies (i.e., McNeil, 1982; McKee, 2001; Woodhead, 2006) appear to be inconsistent with the predictions of prospect and cumulative prospect theory (Kahneman \& Tversky, 1979; Tversky \& Kahneman, 1981/1992), which predict risk aversion when faced with a gain and risk seeking when faced with a loss. Prospect and cumulative prospect theory do not differ when applied to situations with two possible outcomes. One idea offered by the prospect theory is that the cancellation, wherein information identical within each option is ignored, is applied to the information considered when a decision is necessary (Kahneman \& Tversky, 1979). Additionally, differences in small numbers have greater subjective values than identical differences in large values (Tversky \& Kahneman, 1981/1992). When the positive frame is considered from the perspective of prospect theory, the lower probability of living following surgery, as compared to radiation, may have been perceived as a relatively small loss. The surgery option stated that 90 patients live through the end of treatment, and the radiation option stated that 100 people live through the end of treatment. The difference between 90 and 100 people living as a result of the two treatments might not appear significantly different, as both values are relatively large. Therefore, participants may have perceived the 90 people living through surgery and all people living through radiation as nearly identical survival rates. The operation of cancellation would enable people to disregard how many people live through the end of each treatment. The decision maker's focus may have been on the life expectancy following treatment. The greater likelihood of a longer life duration following surgery (e.g., "10 patients live longer than five years"; interval probability format) would be preferable to the lesser likelihood of a longer life duration following radiation (e.g., "5 patients live longer than five years"; interval probability format). Surgery allowed for a greater likelihood of living longer, and was therefore chosen. 
In the negative frame, both options were phrased in terms of loss of life. The low probability of immediate death following radiation might have appeared preferable to the higher probability of immediate death following surgery. The radiation option stated that no patients die by the end of treatment, while the surgery option stated that ten people die by the end of treatment. The comparison of ten versus zero people dying by the end of each treatment may have been perceived as a significant difference, so that cancellation could not occur and participants focused on how many people die by the end of the treatment. The chance of immediate loss of life associated with surgery did not outweigh the long-term benefits of this option. Participants may have focused on avoiding immediate loss, thereby choosing radiation more frequently than surgery.

The information processing approach suggested by Yates (1990) and Sieck and Yates (1997) suggests that increasing the opportunity for decision makers to gain additional information and manipulate presented information might help preclude the framing effect. The process of making the decision in collaboration with a peer should have provided collaborative decision makers with the opportunity to incorporate additional information into their decision making process, as they had the opportunity to benefit from hearing the other person's opinions or advice regarding which decision to choose. However, the individual and collaborative decision makers demonstrated the framing effect, and there was no difference between the proportions of individual and collaborative decision makers who exhibited the framing effect across the three data formats. One possible explanation for this lack of difference is that the collaborators did not discuss various alternatives despite having the opportunity for discussion. Another possibility is that the individual and collaborative decision makers engaged in similar decision making processes. However, assuming that the information processing approach is 
correct, it appears that working collaboratively does not result in sufficient consideration and manipulation of information necessary to preclude the framing effect.

The dual-process reasoning theory proposed by Stanovich and West (2000) would suggest that the framing effect is more likely to occur when decision makers engage in the holistic process, as this process is automatic and dependent of contextual cues such as frame. However, the framing effect is less likely to occur when decision makers engage in the analytic process, as this process involves analyzing and comparing options. Due to the documentation of the framing effect with both individual and collaborative decision makers, it might be concluded that the collaborative decision making process did not induce decision makers to engage in the analytic process, and thereby disregard the frame. The question of whether the collaborative decision makers engaged in analytic processing could be addressed in a future study, as this study did not attempt to yield conclusive evidence regarding this issue.

Proportion of individual and collaborative decision makers exhibiting framing effect

The proportion of individual and collaborative decision makers who exhibited the framing effect was compared to determine if decision makers from one group were more likely to exhibit the framing effect than decision makers from the other group. The proportion of individual decision makers who exhibited the framing effect was not different from the proportion of collaborative decision makers who exhibited the framing effect across data formats. The collaborators were not better at avoiding the framing effect than individuals, as there was no difference in how many people demonstrated the framing effect between the groups. The finding that collaborators did not perform better than the individuals is similar to findings from previous studies comparing memory performance among individuals and dyads (Andersson \& Rönnberg, 1997; Johansson, Andersson, \& Rönnberg, 2000/2005). In these 
previous studies, dyads' performances on memory recall tasks were better than the performance of one individual, but not as good as the performance of the combined scores of two individuals. This study and previous studies (Andersson \& Rönnberg, 1997; Johansson, Andersson, \& Rönnberg, 2000/2005) lend support to the idea that dyads do not necessarily perform better than individuals.

\section{Predicting the framing effect}

Susceptibility to the framing effect could not be predicted by confidence ratings, use of information ratings, gender of decision maker, the decision maker's previous experience with cancer and cancer treatment, or whether the individual made a decision individually or collaboratively. In contrast to this finding, Woodhead (2006) demonstrated that previous experience with cancer or treatment was predictive of susceptibility to the framing effect, in that people were less likely to exhibit the framing effect if they received or provided care for someone or knew someone who'd been diagnosed with cancer. It is unclear why these variables were not predictive of the framing effect among the individual decision makers in the current study. The different findings may possibly be attributable to procedural differences between Woodhead's study and the current study. Woodhead included a debiasing component, and included only individual decision makers. The current study did not include a debiasing component, and included individual and collaborative decision makers. Although the reason regarding why these procedural differences may account for the different findings is currently unknown, these procedural differences may nonetheless contribute to the differences in predicting the framing effect. Alternatively, the relationship between caregiving, cancer experience, and exhibiting the framing effect may not be reliable. 


\section{Confidence ratings within data formats}

The first research question asked if group confidence ratings differed between data formats in which the framing effect was and was not demonstrated. While individual decision makers reported similar confidence ratings regardless of whether the framing effect was exhibited, collaborative decision makers reported higher confidence ratings when the framing effect did not occur as compared to when the framing effect did occur.

Stanovich and West's (2000) holistic and analytic dual-process theory may be useful in interpreting the collaborative decision makers' higher confidence ratings in the data format where the framing effect was not exhibited as compared to the data formats where the framing effect was exhibited. The analytic process should preclude the framing effect, as it would allow for decontextualization of the information. Decontextualization allows for removal of social content, and the use of rules and principles in considering options so that the frame would become irrelevant (Stanovich \& West). Indeed, forcing the consideration of all information has been used as a technique for avoiding the framing effect (e.g., Kim, Goldstein, Hasher, \& Zacks, 2005; Miller \& Fagley, 1991; Sieck \& Yates, 1997; Takemura, 1993/1994). In contrast, the holistic processing approach, which is more reliant on contextual information and heuristics, would be more likely to lead to a framing effect. While collaborators might be expected to discuss the information and engage in the analytic process, they might have nonetheless engaged in the holistic process. If the collaborator's decision making behavior was consistent with the holistic and analytic dual-process theory, the collaborators would have been engaging in the holistic process when the framing effect was demonstrated, and engaging in the analytic process when the framing effect was not demonstrated. The collaborators may have been less confident in their decision while engaging in the holistic approach because they did not carefully consider 
all of the information. Likewise, the collaborators may have been more confident after engaging in the analytic process. Therefore, collaborators using an analytic approach would have been more likely to avoid the framing effect and be more confident in their decisions than those using a more holistic approach.

Use of information ratings within data formats

The second research question asked if use of information ratings differed between data formats in which the framing effect was and was not demonstrated. Participants rated the extent to which they based their decision on the information presented and how thoroughly they considered the information for each data format. The use of information ratings stated by individual and collaborative decision makers were similar when the framing effect was and was not demonstrated. Individual and collaborative decision makers did not perceive that they used the given information differently under the conditions in which the framing effect was demonstrated compared to when it was not.

Confidence rating differences in demonstrating the framing effect

The third research question asked if individual and collaborative decision makers who indicated choice reversals across data formats, thereby demonstrating the framing effect, differed in confidence ratings from those who did not demonstrate the framing effect. This question was important in order to determine if demonstrating the framing effect was related to differences in confidence between participants. Among the individual decision makers, no difference was found for confidence ratings between participants who did and did not demonstrate the framing effect. Likewise, no difference was found for confidence ratings between collaborative decision makers who did and did not demonstrate the framing effect. This finding revealed that selfreports of confidence do not distinguish people who have and have not been influenced by the 
frame. When making real life decisions, a decision maker's statement of his or her confidence regarding a medical treatment may not accurately reflect whether the decision maker was influenced by the frame in which the treatment options were presented. However, as previously noted, there were group differences in collaborator's confidence ratings between data formats in which group choices did and did not demonstrate the framing effect.

\section{Use of information rating differences in demonstrating the framing effect}

The fourth research questions asked if individual and collaborative decision makers who indicated choice reversals across data formats, thereby demonstrating the framing effect, differed in use of information ratings from those who did not demonstrate the framing effect. Use of information assessed the extent to which participants based their decision on the information presented and how thoroughly participants considered the information for each data format. This question was important in order to identify if demonstrating the framing effect was related to how the provided information was used in the decision making task. Among the individual decision makers, no difference was found for use of information ratings between people who did and did not demonstrate the framing effect. Likewise, no difference was found for use of information ratings between collaborative decision makers who did and did not demonstrate the framing effect. When faced with a real-life medical treatment decisions, distinguishing people who have or have not been influenced by the frame of the information may not be possible by asking questions regarding how they used the presented information.

\section{Limitations}

Limitations of the current study should be considered in interpreting the obtained results. A limitation of this study and several other previous studies (i.e., McNeil, 1982; McKee, 2001; Woodhead, 2006) is that the framing effect was examined using only one medical problem (lung 
cancer). Furthermore, this study employed a hypothetical situation. While hypothetical situations have been commonly used in studies regarding the framing effect, different results might be obtained from individuals who are faced with real life medical treatment decisions. Additionally, this study limited the number of treatment choices to two: surgery and radiation. This study's forced choice between surgery or radiation may decrease the generalizability of the findings, as cancer patients may have other alternatives for treatment (i.e., chemotherapy or a combination of treatments).

The current study employed a between-subjects design. While this design prevented participants from becoming aware of the framing differences among the scenarios, the design also had some limitations. This study examined a person's susceptibility to the framing effect by examining changes in decisions across frames, yet a within-subject design would have allowed for direct comparison of a participant's decision in the positive and negative frames. However, use of a within-subject design (cf., McKee, 2001), also has limitations. Participants may become aware of the framing differences in scenarios during a within-subject design, which could influence treatment decision. Additionally, participant fatigue may occur more frequently with the increased length of testing session. While the between-subject design was chosen for this study to prevent participant's awareness of the presentation of frame, this design nevertheless creates different limitations than those that would have occurred using a within-subject design.

The participant sample of this study may be viewed as a limitation. Participants were predominantly females living in small metropolitan areas. There was little ethnic diversity within the sample, which is reflective of the population from which participants were recruited. While the sample was limited in diversity, the extent to which this lack of diversity limits the findings is uncertain. 


\section{Conclusions}

The framing effect is reliable among individual decision makers, and has now been demonstrated among collaborative decision makers. Furthermore, collaborators appear equally susceptible as individuals to demonstrating the framing effect. While older adults often discuss options with another person when faced with a real life decision (Strough et al., 2003), collaboration appears insufficient in preventing the framing effect.

The framing effect could not be predicted by the variables tested in this study. Additionally, individual decision makers' group confidence and use of information ratings did not differ when the framing effect was or was not demonstrated. Additionally, collaborative decision makers' group use of information ratings did not differ when the framing effect was or was not demonstrated. However, the collaborative decision makers' group confidence ratings were lower in data formats in which the framing effect occurred as compared to the format in which it did not occur. The results from the research questions might indicate that individual and collaborative decision makers engage in different decision making processes.

When the framing effect was examined by looking at personal treatment preference reversal across data formats, neither the confidence nor use of information ratings distinguished individual decision makers who demonstrated the framing effect from those who did not. Likewise, neither the confidence nor use of information ratings distinguished collaborative decision makers who demonstrated the framing effect from those who did not. When people are faced with real-life decisions, the decision maker's report of confidence and their use of the information may not be indicative of whether the person was influenced by the frame of the information. This appears to be true regardless of whether the decision makers decide individually or in collaboration with a peer. 


\section{Future Directions}

There are several different directions for future research investigating the framing effect in medical decision making. First, researchers should examine the framing effect when individuals are faced with real-life medical treatment decisions to determine the generalizability of decisions made with hypothetical problems. Similarly, the generalizability of collaborative decision making should be examined with real-life medical treatment decisions.

Methods for precluding the framing effect among collaborative decision makers should be investigated in future studies. Previous studies have examined methods for precluding the framing effect for individual decision makers (i.e., Kim et al., 2005; Mashat 2004; McElroy \& Seta, 2003; Miller \& Fagley, 1991; Takemura, 1992/1993/1994; Woodhead, 2006). In the current study, collaborative decision making was initially perceived as a possible method for precluding the framing effect. However, the results from this study indicate that collaborative decision makers demonstrated the framing effect in more data formats than the individual decision makers. In light of this evidence, it is important to determine if methods that have been successful for precluding the framing effect among individual decision makers are successful for precluding the framing effect among collaborative decision makers. Methods for precluding the framing effect among collaborative decision makers may be useful for preventing the framing effect when people are faced with making a real treatment decision.

The effects of framing on collaborative decision makers of younger age groups could yield information regarding collaborator's susceptibility to the framing effect across the lifespan. Younger adults are susceptible to the framing effect (Kim, Goldstein, Hasher, \& Zacks, 2005; Rönnlund, Karlsson, Laggnäs, Larsson, \& Lindström, 2005; Woodhead, 2006), but the framing effect has only been examined among dyads consisting of older adults. Based on the information 
obtained in this study, young adult collaborative decision makers would be expected to demonstrate the framing effect in as many, if not more, data formats as young adult individual decision makers.

The relationship between confidence and demonstrating the framing effect could be examined in a future study. The current study found that collaborative decision makers' confidence ratings differ when the framing effect was or was not demonstrated. However, the reason for this change in confidence is unknown. Several questions can be asked regarding the difference in the collaborative decision maker's confidence levels. First, why are they succumbing to the framing effect when they are less confident? Or, perhaps, why are they less confident after succumbing to the framing effect? A future study could attempt to clarify how confidence is related to the demonstration of the framing effect among collaborators.

Future research aimed at discovering which aspects of the questions are most salient in the decision making process could yield answers regarding how the prospect theory does or does not predict the pattern of results obtained here. This study demonstrated a reversed pattern of risk seeking and risk avoidance choices across frames as compared to studies documenting the framing effect in non-medical decision making tasks (e.g., LeBoeuf \& Shafir, 2003; Miller \& Fagley, 1991; Takemura, 1993/1994; Tversky \& Kahneman, 1981). While these previous studies found risk seeking behavior in positive frames and risk avoidance behavior in negative frames, this study found risk avoidance behavior in the positive frame and risk seeking behavior in the negative frame. The reversed pattern found in this study and previous studies employing a personal decision making task (e.g., LeBoeuf and Shafir, 2003; McKee, 2001; McNeil, 1982; Woodhead, 2006) should be further examined. Future studies could investigate why this reversal 
is found with personal medical treatment decision making, but not with other types of decision making tasks.

Stanovich and West's (2000) dual-process theory could be tested more directly by determining when the decision makers engage in either the analytic or holistic process. This type of study could yield information regarding how the dual-process theory accounts for the framing effect among individual and collaborative decision makers. This topic could be investigated through questioning decision makers in order to ascertain if they typically engage in the analytic or holistic process (c.f., McElroy \& Seta, 2003)

This study highlighted that individual and collaborative decision makers are influenced differently by the framing of choices. Future studies investigating the framing effect should attempt to include collaborators as well as individuals, as results for individual decision makers may not be generalizable to collaborators. 


\section{References}

Andersson, J. \& Rönnberg, J. (1997). Cued memory collaboration: Effects of friendship and type of retrieval cue. European Journal of Cognitive Psychology, 9(3), 273-287.

Arkes, H.R., \& Blumer, C. (1985). The psychology of sunk cost. Organizational Behavior and Human Decision Processes, 35, 124-140.

Armstrong, K., Schwartz, J.S., Fitzgerald, G., Putt, M., \& Ubel, P.A. (2002). Effect of framing as gain versus loss on understanding and hypothetical treatment choices: Survival and mortality curves. Medical Decision Making, 22, 76-83.

Baron, J. (2000). Descriptive theory of choice under certainty. In Thinking and deciding, $3^{\text {rd }}$ edition. (pp. 245-276). Cambridge, UK: Cambridge University Press.

Bernstein, L.M., Chapman, G.B., \& Elstein, A.S. (1999). Framing effects in choices between multioutcome life-expectancy lotteries. Medical Decision Making, 19, 324-338.

Dixon, R., \& Gould, O. (1998). Younger and older adults collaborating on retelling everyday stories. Applied Developmental Science, 2(3), 160-171.

Gould, O., Trevithick, L., \& Dixon, R. (1991). Adult age differences in elaborations produced during prose recall. Psychology and Aging, 6(1), 93-99.

Johansson, O., Andersson, J., \& Rönnberg, J. (2000). Do elderly couples have a better prospective memory than other elderly people when they collaborate? Applied Cognitive Psychology, 14, 121-133.

Johansson, O., Andersson, J., \& Rönnberg, J. (2005). Compensating strategies in collaborative remembering in very old couples. Scandinavian Journal of Psychology, 46, 349-359.

Kahneman, D., \& Tversky, A. (1979). Prospect theory: An analysis of decision under risk. Econometrica, 47, 263-291. 
Kim, S., Goldstein, D., Hasher, L., \& Zacks, R.T. (2005). Framing effects in younger and older adults. Journal of Gerontology, 60B (4), 215-218.

Krishnamurthy, P., Carter, P., \& Blair, E. (2001). Attribute framing and goal framing effects in health decisions. Organizational Behavior and Human Decision Processes, 85(2), 382399.

Kühberger, A. (1998). The influence of framing on risky decisions: A meta-analysis. Organizational Behavior and Human Decision Processes, 75(1), 23-55.

Kühberger, A., Schulte-Mecklenbeck, M., \& Perner, J. (1999). The effects of framing, reflection, probability, and payoff on risk preference in choice tasks. Organizational Behavior and Human Decision Processes, 78(3), 204-231.

Kühberger, A., Schulte-Mecklenbeck, M., \& Perner, J. (2002). Framing decisions: Hypothetical and real. Organizational Behavior and Human Decision Processes, 89, 1162-1175.

Lauriola, M., Russo, P.M., Lucidi, F., Violani, C., \& Levin, I.P. (2005). The role of personality in positively and negatively framed risky health decisions. Personality and Individual Differences, 38, 45-59.

Lawton, M., Moss, M., Fulcomer, M., \& Kleban, M. (1982). A research and service oriented multilevel assessment instrument. Journal Of Gerontology, 37(1), 91-99.

LeBoeuf, R.A., \& Shafir, E. (2003). Deep thoughts and shallow frames: On the susceptibility to framing effects. Journal of Behavioral Decision Making, 16(2), 77-92.

Levin, I.P. \& Chapman, D.P. (1990). Risk taking, frame of reference, and characterization of victim groups in AIDS treatment decisions. Journal of Experimental Social Psychology, $26,421-434$. 
Levin, I.P. \& Gaeth, G.J. (1988). How consumers are affected by the framing of attribute information before and after consuming the product. Journal of Consumer Research, 15, 374-378.

Levin I.P., Gaeth, G.J., Schreiber, J., \& Lauriola, M. (2002). A new look at framing effects: Distribution of effect sizes, individual differences, and independence of types of effects. Organizational Behavior and Human Decision Processes, 88(1), 411-429.

Levin, I.P., Schneider, S.L., \& Gaeth, G.J. (1998). All frames are not created equal: A typology and critical analysis of framing effects. Organizational Behavior and Human Decision Processes, 76(2), 149-188.

Markowitz, H. (1952). The utility of wealth. Journal of Political Economy, 60, 151-158.

Mashat, S. (2004) Framing effect debiasing in medical decision making. Unpublished Honors thesis, West Virginia University.

Mayhorn, C.B., Fisk, A.D., \& Whittle, J.D. (2002). Decisions, decisions: Analysis of age, cohort, and time of testing on framing of risky decision options. Human Factors, 44(4), $515-521$.

McElroy, T. \& Seta, J.J. (2003). Framing effects: An analytic-holistic perspective. Journal of Experimental Social Psychology, 39, 610-617.

McKee, D.R. (2001). The effects of framing on younger and older adults' medical decision making. Unpublished doctoral dissertation, West Virginia University.

McNeil, B.J., Pauker, S.G., Sox, H.C., \& Tversky, A. (1982). On the elicitation of preferences for alternative therapies. The New England Journal of Medicine, $306,1259-1262$. 
Meegan, S., \& Berg, C. (2002). Contexts, functions, forms, and processes of collaborative everyday problem solving in older adulthood. International Journal of Behavioral Development, 26(1), 6-15.

Miller, P.M, \& Fagley, N.S. (1991). The effects of framing, problem variations, and providing rationale on choice. Personality and Social Psychology Bulletin, 17(5), 517-522.

Moxey, A., O’Connell, D., McGettigan, P., \& Henry, D. (2003). Describing treatment effects to patients: How they are expressed makes a difference. Journal of General Internal Medicine, 18, 948-959.

Nelson, W., Stefanek, M., Peters, E., \& McCaul, K.D. (2005). Basic and applied decision making in cancer control. Health Psychology, 24(4 suppl.), S3-S8.

Paese, P., Bieser, M., \& Tubbs, M. (1993). Framing effects and choice shifts in group decision making. Organizational Behavior and Human Decision Processes, 56(1), 149-165.

Pinon, A. \& Gambara, H. (2005). A meta-analytic review of framing effect: risky, attribute, and goal framing. Psicothema, 17(2), 325-331.

Rönnlund, M., Karlsson, E., Laggnäs, E., Larsson, L., \& Linström, T. (2005). Risky decision making across three arenas of choice: Are younger and older adults differently susceptible to framing effects? The Journal of General Psychology, 132(1), 81-92.

Rothman, A.J., Martino, S.C., Bedell, B.T., Detweiler, J.B., \& Salovey, P. (1999). The systematic influence of gain- and loss-framed messages on interest in and use of different types of health behavior. Personality and Social Psychology Bulletin, 25(11), $1355-$ 1369.

Saczynski, J., Margrett, J., \& Willis, S. (2004). Older adults' strategic behavior: Effects of individual versus collaborative cognitive training. Educational Gerontology, 30(7), 587. 
Schuller, K.L. (2006). The framing effect and breast cancer treatment options: Do individual characteristics play a role? Unpublished doctoral dissertation, West Virginia University.

Schulz-Hardt, S., Frey, D., Lüthgens, C., \& Moscovici, S. (2000). Biased information search in group decision making. Journal of Personality and Social Psychology, 78(4), 655-669.

Sieck, W. \& Yates, J.F. (1997). Exposition effects on decision making: Choice and confidence in choice. Organizational Behavior and Human Decision Processes, 70(3), 207-219.

Smith, C. M., Tindale, R.S., \& Steiner, L. (1998). Investment decisions by individuals and groups in 'sunk cost' situations: The potential impact of shared representations. Group Processes and Intergroup Relations, 2, 175-189.

Soane, E., \& Chmiel, N. (2005). Are risk preferences consistent? The influence of decision domain and personality. Personality and Individual Differences, 38, 1781-1791.

Stanovich, K.E. \& West, R.F. (2000). Individual differences in reasoning: Implications for the rationality debate? Behavioral and Brain Sciences, 23, 645-726.

Strough, J., Patrick, J.H., Swenson, L.M., Cheng, S. \& Barnes, K.A. (2003). Collaborative everyday problem solving: Interpersonal Relationships and Problem Dimensions. The International Journal of Aging and Human Development, 56(1), 43-66.

Takemura, K. (1992). Effect of decision time on framing of decision: A case of risky choice behavior. Psychologia, 35, 180-185.

Takemura, K. (1993). The effect of decision frame and decision justification on risky choice. Japanese Psychological Research, 35(1), 36-40.

Takemura, K. (1994). Influence of elaboration on the framing of decision. The Journal of Psychology, 128(1), 33-39. 
Tversky, A. \& Kahneman, D. (1981). The framing of decisions and the psychology of choice. Science, 211, 453-458.

Tversky, A. \& Kahneman, D. (1992). Advances in prospect theory: Cumulative representation of uncertainty. Journal of Risk and Uncertainty, 5, 297-323.

Von Neumann, J. \& Morgenstern, O. (1944). Theory of games and economic behavior. New York: John Wiley \& Sons, Inc.

Whyte, G. (1993). Escalating commitment in individual and group decision making: A prospect theory approach. Organizational Behavior and Human Decision Processes, 54, 430-455.

Woodhead, E. L. (2006). Debiasing the framing effect in younger and older adults' medical decision-making. Unpublished master's thesis, West Virginia University.

Yates, J.F. (1990). Judgment and decision making. Englewood Cliffs, New Jersey: Prentice Hall, Inc. 
Appendix A

\section{Participant Instructions}

The following pages contain specific information about cancer treatments at several Chicago area hospitals. Each hospital has its own doctors and policies regarding patient care, approaches to treatment, and different survival rates for the various types of treatment. For each hospital, please indicate whether you prefer surgery or radiation therapy. Below are general descriptions of the treatments.

Surgery for lung cancer involves an operation on the lungs. Most patients are in the hospital for two to three weeks and have some pain around their incisions; they spend a month or so recuperating at home. After that they generally feel fine.

Radiation therapy for lung cancer involves the use of radiation to kill the tumor and requires coming to the hospital about four times a week for six weeks. Each treatment takes a few minutes, and during the treatment patients lie on a table as if they were having an x-ray. During the course of treatment, some patients develop nausea and vomiting, but by the end of six weeks they generally feel fine.

Thus, after the initial six weeks, patients treated with either surgery or radiation therapy feel about the same.

Please answer the following questions in the order that they appear. Do not read ahead. Once you've answered a question, do not return to it. 


\section{Appendix B}

(Mortality Frame, Cumulative Probability)

\section{$\underline{\text { Hospital } 1}$}

- Of 100 patients having surgery, 10 die during the treatment, 32 die by one year, and 66 die by 5 years.

- Of 100 patients having radiation therapy, no patients die during treatment, 23 die by one year, and 78 die by 5 years. 
(Mortality Framed, Interval Probability)

\section{$\underline{\text { Hospital } 2}$}

- Of 100 patients having radiation therapy, no patients die by the end of treatment, 32 die in the time interval between treatment and one year, 55 die in the interval between one and five years, and 22 die sometime after five years.

- Of 100 patients having surgery, 10 patients die by the end of treatment, 22 patients die in the interval between treatment and one year, 34 patients die in the interval between one and five years, and 34 patients die sometime after five years. 
(Mortality Framed, Life Expectancy)

$\underline{\text { Hospital } 3}$

- Of 100 patients having radiation therapy, no patients die during treatment. The patients who survive treatment have an average life expectancy of 4.7 years.

- Of 100 patients having surgery, 10 percent of the patients die during treatment. The patients who survive surgery have an average life expectancy of 6.8 years. 
(Survival Framed, Cumulative Probability)

\section{$\underline{\text { Hospital } 1}$}

- Of 100 patients having radiation therapy, all patients live through the treatment, 77 patients live for more than one year, and 22 patients live for more than five years.

- Of 100 patients having surgery, 90 patients live through treatment, 68 patients live for more than one year, and 34 patients live for more than five years. 
(Survival Framed, Interval Probability)

\section{$\underline{\text { Hospital } 2}$}

- Of 100 patients having surgery, 90 patients live until the end of treatment, 78 patients live through treatment but less than one year, 44 patients live for one to five years, and 10 patients live longer than five years.

- Of 100 patients having radiation therapy, all patients live through the end of treatment, 77 live through the treatment but less than one year, 22 patients live for one to five years, and 5 patients live for more than five years. 
(Survival Framed, Life Expectancy)

\section{$\underline{\text { Hospital } 3}$}

- Of 100 patients having surgery, 90 percent of the patients live through treatment. The patients who survive surgery have an average life expectancy of 6.8 years.

- Of 100 patients having radiation therapy, all patients live through treatment. The patients who survive radiation therapy have an average life expectancy of 4.7 years. 


\section{Appendix C}

(Individual decision maker's instructions)

You will be asked to choose one of the two treatments, surgery or radiation. Spend as much time as needed considering whether to choose surgery or radiation.

(Collaborative decision maker instructions)

You will be asked to choose one of the two treatments, surgery or radiation. Spend as much time as needed discussing with your partner whether to choose surgery or radiation.

(Collaborative Non-decision maker's instructions)

Your partner will be asked to choose one of the two treatments, surgery or radiation. Spend as much time as needed discussing with your partner whether to choose surgery or radiation. 


\section{Appendix D}

(Treatment Choice Questionnaire)

Which cancer treatment would you prefer? Circle one:

Surgery

Radiation Therapy 


\section{Appendix E}

(Confidence Rating--Decision Maker)

Please indicate how confident you are that you made the best choice:

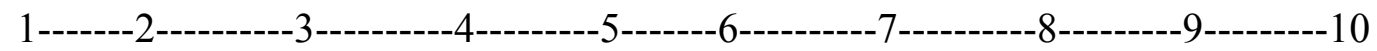

Not at all confident

Very Confident

Please indicate how comfortable you are with your choice:

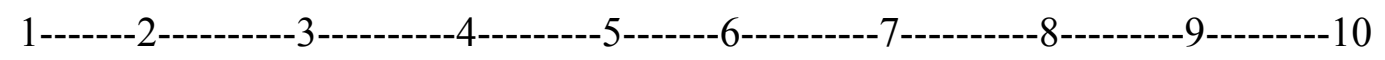

Not at all

Very Comfortable

Please indicate how worried you are that you did not make the best choice:

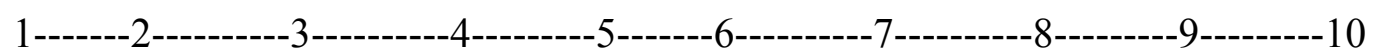

Not at all worried

Very Worried 


\section{Appendix F \\ (Confidence Rating—Non-Decision Maker)}

Please indicate how confident you are that your partner made the best choice:

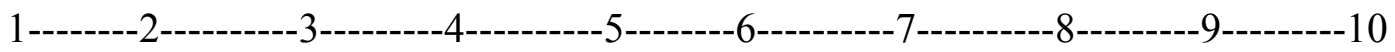

Not at all confident

Very Confident

Please indicate how comfortable you are with your partner's choice:

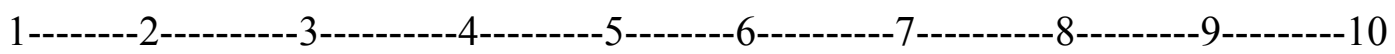

Not at all

Very Comfortable

Please indicate how worried you are that your partner did not make the best choice:

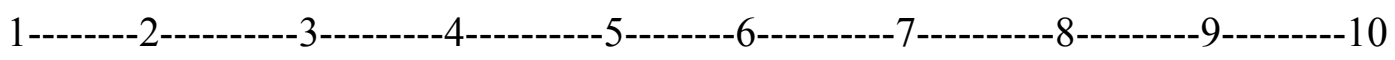

Not at all worried

Very Worried 
Appendix G

(Use of Information-Decision Maker)

To what extent was your decision based on the information presented?

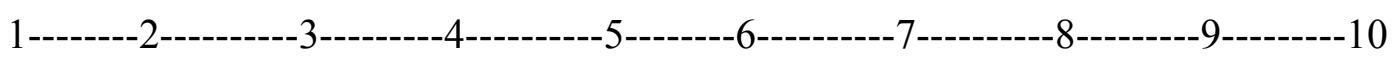

Not at all

Very much

How thoroughly did you consider the information presented?

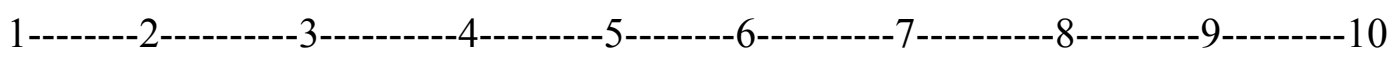

Not at all

Very much 


\section{Appendix $\mathrm{H}$}

\section{(Use of Information-Non-Decision Maker)}

If you were making the medical decision, to what extent would your decision be based on the information presented?

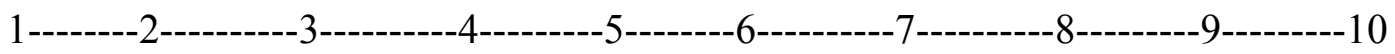

Not at all

Very much

How thoroughly did you consider the information presented?

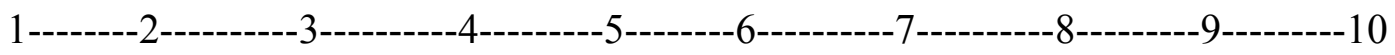

Not at all

Very much 


\section{Appendix I}

Demographic Questionnaire

1. Age: __ Gender: (circle one) Male Female

2. Years of Education:

3. Marital Status: (circle one)

Single $\quad$ Married Separated Divorced Widowed

4. If married/separated/divorced/widowed:

Number of Years Married to current/most recent spouse:

Number of Marriages:

5. Ethnicity/race: (circle one)

Caucasian (White)

Pacific Islander

African American (Black)

Asian American

Hispanic

Native American (American Indian/Alaskan

Native)

Other:

6. What is your current occupation or the occupation you pursued for the majority of your adult life?

7. Have you ever been diagnosed with a chronic illness, that is, a disease that is ongoing or longlasting (e.g., heart disease, diabetes, arthritis)?

Yes No

8. How long has it been since you were examined by a doctor?

9. How would you rate your overall health at the present time?
(1) Excellent
(2) Good
(3) Fair
(4) Poor

10. Is your health now better, about the same, or not as good as it was three years ago?:
(1) Better
(2) About the Same
(3) Not as Good

11. Do your health problems stand in the way of your doing the things you want to do?
(1) Not at All
(2) A Little
(3) A Great Deal 
12. Would you say your health is better, about the same, or not as good as most people your age?:
(1) Better
(2) The Same
(3) Not as Good.

13. Do you smoke? Yes No

If yes, how many years have you smoked?

14. Have you ever been diagnosed with any type of cancer? Yes No If yes, what type of cancer?

15. Has someone close to you ever been diagnosed with any type of cancer? Yes No If yes, what type of cancer?

Please indicate your relationship to this person:

16. Have you ever received or provided care for someone? Yes No

If yes, were you the care receiver or provider? (circle one)

\section{Receiver Provider}

How long were you the care receiver or provider?

17. Have you ever discussed the experience of cancer with someone who had cancer?

Yes No

If so, please rate how involved you were in this person's life during their experience with cancer:

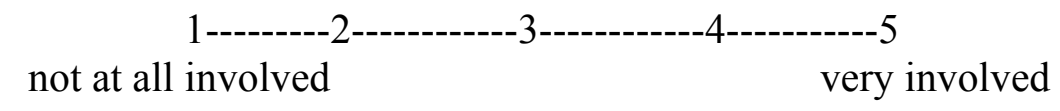

18. Have you ever discussed the treatment of cancer with someone who had cancer?

Yes No

If so, please rate how involved you were in this person's life during their treatment of cancer:

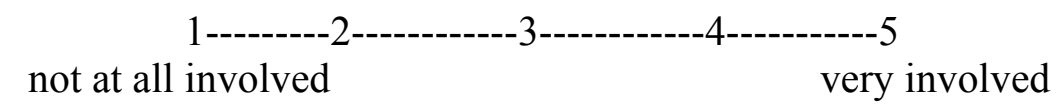

